\title{
On Interval Observer Design for a Class of Continuous-Time LPV Systems
}

\author{
Stanislav Chebotarev* Denis Efimov**,* Tarek Raïssi ${ }^{* * *}$ \\ Ali Zolghadri ${ }^{* * * *}$ \\ * Department of Control Systems and Informatics, Saint Petersburg \\ State University of Information Technologies Mechanics and Optics \\ (ITMO), Kronverkskiy av. 49, Saint Petersburg, 197101, Russia, \\ freest5@gmail.com \\ ** Non-A team of INRIA-LNE, Parc Scientifique de la Haute Borne, \\ 40 avenue Halley, Bt.A Park Plaza, 59650 Villeneuve d'Ascq, France, \\ Denis.Efimov@inria.fr \\ *** Conservatoire National des Arts et Mtiers (CNAM), Cedric - \\ laetitia 292, Rue St-Martin, case 2D2P10, 75141 Paris Cedex 03, \\ tarek.raissi@cnam.fr \\ **** University of Bordeaux, IMS-lab, Automatic control group, 351 \\ cours de la libration, 33405 Talence, France, \\ Ali.Zolghadri@ims-bordeaux.fr
}

\begin{abstract}
This work is devoted to interval observer design for Linear Parameter-Varying (LPV) systems under assumption that the vector of scheduling parameters is not available for measurements. Stability conditions are expressed in terms of matrix inequalities, which can be solved using standard numerical solvers. Robustness and estimation accuracy with respect to model uncertainty is analyzed using $L_{\infty} / L_{1}$ framework. Two solutions are proposed for nonnegative systems and for a generic case. The efficiency of the proposed approach is demonstrated through computer simulations.
\end{abstract}

\section{INTRODUCTION}

The problem of unmeasurable (partially or completely) state vector estimation for nonlinear systems is very challenging and can find many applications Meurer et al. [2005], Fossen and Nijmeijer [1999], Besanon [2007]. Frequently, in the nonlinear case, observer or controller design is based on transformation of the system into canonical forms, which can be an obstruction in practice. That is why the class of LPV systems became very popular in applications: a wide class of nonlinear systems can be presented in the LPV form, while partial linearity of LPV models allows one to apply several frameworks developed for linear systems Shamma [2012], Marcos and Balas [2004], Shamma and Cloutier [1993], Tan [1997]. In some situations due to the presence of uncertainty (parametric or/and signal) the design of a conventional estimator, converging in the noise-free case to the ideal value of the state, is complicated. However, an interval estimation may still remain feasible. By interval estimation we understand an observer that, using input-output information, evaluates the set of admissible values (interval) for the state at each instant of time.

\footnotetext{
^ This work is partially supported by financial support of FGP "Research and scientific-pedagogical personnel of innovative Russia" for 2009 - 2013 years (the agreement 14.B37.21.0875), by EU Interreg IV A 2 Mers Seas Zeeën Cross-border Cooperation Programme under SYSIASS project 06-020, and by Ministry of Higher Education and Research, Nord-Pas de Calais Regional Council and FEDER through the "Contrat de Projets Etat Region (CPER) CIA 2007-2013".
}

There are several approaches to design interval observers Jaulin [2002], Kieffer and Walter [2004], Olivier and Gouzé [2004], Moisan et al. [2009]. This paper continues the framework of interval observer design based on the monotone system theory Olivier and Gouzé [2004], Moisan et al. [2009], Raïssi et al. [2010, 2012], Efimov et al. [2012]. One of the most restrictive assumptions for the interval observer design deals with cooperativity of the interval estimation error dynamics, which was recently relaxed in Mazenc and Bernard [2011], Raïssi et al. [2012], Combastel [2013]. In those studies, it has been shown that under some mild conditions applying similarity transformation, a Hurwitz matrix could be transformed to a Hurwitz and Metzler one (cooperative). In order to apply the approach of interval observer design to the systems with non-constant matrices dependent on measurable input-output signals and time, an extension of the result from Raïssi et al. [2012] has been presented in Efimov et al. [2013a], where a constant similarity transformation matrix representing a given interval of matrices to an interval of Metzler matrices is needed. Thus this method can be used to design interval observers for LPV systems only with a measurable vector of scheduling parameters (as it is noted in Efimov et al. [2013a]).

The objective of this note is to propose some new results on interval observer design for LPV systems with an unmeasurable vector of scheduling parameters.

The paper is organized as follows. Some basic facts from the theory of interval estimation are given in Section 2. The 
main results are described in Section 3. Two examples of computer simulations are presented in Section 4.

\section{PRELIMINARIES}

Euclidean norm for a vector $x \in \mathbb{R}^{n}$ will be denoted as $|x|$, and for a measurable and locally essentially bounded input $u: \mathbb{R}_{+} \rightarrow \mathbb{R}\left(\mathbb{R}_{+}=\{\tau \in \mathbb{R}: \tau \geq 0\}\right)$ the symbol $\|u\|_{\left[t_{0}, t_{1}\right]}$ denotes its $L_{\infty}$ norm:

$$
\|u\|_{\left[t_{0}, t_{1}\right]}=e s s \sup \left\{|u(t)|, t \in\left[t_{0}, t_{1}\right]\right\},
$$

if $t_{1}=+\infty$ then we will simply write $\|u\|$. We will denote as $\mathcal{L}_{\infty}$ the set of all inputs $u$ with the property $\|u\|<\infty$. Denote the sequence of integers $1, \ldots, k$ as $\overline{1, k}$. The symbols $I_{n}, E_{n \times m}$ and $E_{p}$ denote the identity matrix with dimension $n \times n$, the matrix with all elements equal 1 with dimension $n \times m$ and $p \times 1$ respectively. For a matrix $A \in \mathbb{R}^{n \times n}$ the vector of its eigenvalues is denoted as $\lambda(A)$, and $\|A\|_{2}=\sqrt{\max _{i=\overline{1, n}} \lambda_{i}\left(A^{\mathrm{T}} A\right)}$ (the induced $L_{2}$ matrix norm).

For two vectors $x_{1}, x_{2} \in \mathbb{R}^{n}$ or matrices $A_{1}, A_{2} \in \mathbb{R}^{n \times n}$, the relations $x_{1} \leq x_{2}$ and $A_{1} \leq A_{2}$ are understood elementwise. The relation $P \prec 0(\bar{P} \succ 0)$ means that the matrix $P \in \mathbb{R}^{n \times n}$ is negative (positive) definite. Given a matrix $A \in \mathbb{R}^{m \times n}$, define $A^{+}=\max \{0, A\}, A^{-}=A^{+}-A$ (similarly for vectors) and denote the matrix of absolute values of all elements by $|A|=A^{+}+A^{-}$.

Lemma 1. Let $x \in \mathbb{R}^{n}$ be a vector variable, $x \leq x \leq \bar{x}$ for some $\underline{x}, \bar{x} \in \mathbb{R}^{n}$, and $A \in \mathbb{R}^{m \times n}$ be a constant matrix, then

$$
A^{+} \underline{x}-A^{-} \bar{x} \leq A x \leq A^{+} \bar{x}-A^{-} \underline{x} .
$$

Proof. For $\underline{x} \leq x \leq \bar{x}$ we have $A x=\left(A^{+}-A^{-}\right) x$ that gives the required relations.

Lemma 2. Let $\underline{A} \leq A \leq \bar{A}$ for some $\underline{A}, \bar{A}, A \in \mathbb{R}^{n \times n}$ and $\underline{x} \leq x \leq \bar{x}$ for $\underline{x}, \bar{x}, x \in \overline{\mathbb{R}}^{n}$, then

$$
\begin{gathered}
\underline{A}^{+} \underline{x}^{+}-\bar{A}^{+} \underline{x}^{-}-\underline{A}^{-} \bar{x}^{+}+\bar{A}^{-} \bar{x}^{-} \leq A x \\
\leq \bar{A}^{+} \bar{x}^{+}-\underline{A}^{+} \bar{x}^{-}-\bar{A} \underline{x}^{+}+\underline{A}^{-} \underline{x}^{-} .
\end{gathered}
$$

Proof. By definition $A x=\left(A^{+}-A^{-}\right)\left(x^{+}-x^{-}\right)=$ $A^{+} x^{+}-A^{+} x^{-}-A^{-} x^{+}+A^{-} x^{-}$, where all terms are elementwise positive, which gives the required relations.

In the case of $\bar{A}^{+}=\underline{A}^{+}=A^{+}$and $\bar{A}^{-}=\underline{A}^{-}=A^{-}$ the result of Lemma 1 follows Lemma 2. Furthermore, if $-\bar{A}=\underline{A} \leq 0 \leq \bar{A}$, then the inequality (2) can be simplified: $-\bar{A}\left(\bar{x}^{+}+\underline{x}^{-}\right) \leq A x \leq \bar{A}\left(\bar{x}^{+}+\underline{x}^{-}\right)$.

A matrix $A \in \mathbb{R}^{n \times n}$ is called Hurwitz if all its eigenvalues have negative real parts, it is called Metzler if all its elements outside the main diagonal are nonnegative. Any solution of the linear system

$$
\begin{gathered}
\dot{x}=A x+B \omega(t), \omega: \mathbb{R}_{+} \rightarrow \mathbb{R}_{+}^{q}, \\
y=C x+D \omega(t),
\end{gathered}
$$

with $x \in \mathbb{R}^{n}, y \in \mathbb{R}^{p}$ and a Metzler matrix $A \in \mathbb{R}^{n \times n}$, is elementwise nonnegative for all $t \geq 0$ provided that $x(0) \geq 0$ and $B \in \mathbb{R}_{+}^{n \times q}$ Farina and Rinaldi [2000], Smith [1995]. The output solution $y(t)$ is nonnegative if $C \in \mathbb{R}_{+}^{p \times n}$ and $D \in \mathbb{R}_{+}^{p \times q}$. Such dynamical systems are called cooperative (monotone) and nonnegative if only initial conditions in $\mathbb{R}_{+}^{n}$ are considered Farina and Rinaldi [2000], Smith [1995].

The $L_{1}$ and $L_{\infty}$ gains for stabilization of nonnegative systems (3) have been studied in Briat [2011], Ebihara et al. [2011], for this kind of systems these gains are interrelated.

Lemma 3. Briat [2011], Ebihara et al. [2011] Let the system (3) be nonnegative (i.e. $A$ is Metzler, $B \geq 0, C \geq 0$ and $D \geq 0)$, then it is asymptotically stable if and only if there exist $\lambda \in \mathbb{R}^{n}, \lambda>0$ and a scalar $\gamma>0$ such that the following LMI is feasible:

$$
\left[\begin{array}{c}
A^{\mathrm{T}} \lambda+C^{\mathrm{T}} E_{p} \\
B^{\mathrm{T}} \lambda-\gamma E_{q}+D^{\mathrm{T}} E_{p}
\end{array}\right]<0 .
$$

Moreover, in this case the $L_{1}$ gain of the transfer $\omega \rightarrow y$ is lower than $\gamma$.

\section{MAIN RESULT}

Consider an LPV system:

$$
\dot{x}=\left[A_{0}+\Delta A(\rho(t))\right] x+b(t), y=C x+v(t), t \geq 0,
$$

where $x \in \mathbb{R}^{n}$ is the state, $y \in \mathbb{R}^{p}$ is the output available for measurements, $\rho(t) \in \Pi \subset \mathbb{R}^{r}$ is the vector of scheduling parameters with a known $\Pi, \rho \in \mathcal{L}_{\infty}^{r}$. The values of the scheduling vector $\rho$ are not available for measurements, and only the set of admissible values $\Pi$ is given. The matrices $A_{0} \in \mathbb{R}^{n \times n}$ and $C \in \mathbb{R}^{p \times n}$ are known, the matrix function $\Delta A: \Pi \rightarrow \mathbb{R}^{n \times n}$ is piecewise continuous and also known for a given value of $\rho$. The signals $b: \mathbb{R}_{+} \rightarrow \mathbb{R}^{n}$ and $v: \mathbb{R}_{+} \rightarrow \mathbb{R}^{p}$ are the external input and measurement noise respectively, the exact current values of $b(t)$ and $v(t)$ are not available.

\subsection{Problem statement}

For brevity of presentation we will use the following assumptions in this work.

Assumption 1. $x \in \mathcal{L}_{\infty}^{n} ; \underline{b}(t) \leq b(t) \leq \bar{b}(t)$ and $|v(t)| \leq V$ for all $t \geq 0$ and some known $\underline{b}, \bar{b} \in \overline{\mathcal{L}}_{\infty}^{n}$ and $V>0$.

Assumption 2. $\underline{\Delta A} \leq \Delta A(\rho) \leq \overline{\Delta A}$ for all $\rho \in \Pi$ and some known $\underline{\Delta A, \overline{\Delta A}} \in \mathbb{R}^{n \times n}$.

Therefore, it is assumed that the state $x(t)$ of the system (4) is bounded, the measurement noise $v(t)$ has an upper bound $V$ and the input $b(t)$ belongs to a known bounded interval $[\underline{b}(t), \bar{b}(t)]$ for all $t \in \mathbb{R}_{+}$. It is also assumed that the matrix $\Delta A(\rho)$ belongs to the interval $[\underline{\Delta A}, \overline{\Delta A}]$ for all $t \geq 0$, which is easy to compute for a given set $\Pi$ (in a polytopic case, for example).

Th objective of this work is to design an interval observer for the system (4).

An interval observer for such a class of systems has been proposed in Efimov et al. [2013b] in the context of system stabilization. In that work stability of the interval estimation error dynamics is not established on the observer design step. More precisely, in that work the stability of the interval observer was ensured by a proper choice of control input. In the present work, only the interval observer is designed and some special conditions on the observer gain are needed to guarantee stability in 
contrast to Efimov et al. [2013b]. Another interval observer for such an LPV system has been formulated in Raïssi et al. [2010], however the conditions of cooperativity of the estimation error dynamics and its stability are restrictive. For the case of measured vector $\rho(t)$ in (4), an interval observer was proposed in Efimov et al. [2013a] using a static transformation of coordinates.

Before introduction of interval observer equations note that for a matrix $L \in \mathbb{R}^{n \times p}$ the system (4) can be rewritten as follows:

$$
\dot{x}=\left[A_{0}-L C\right] x+\Delta A(\rho(t)) x+L[y-v(t)]+b(t),
$$

and according to Lemma 2 and Assumption 2 we have for all $\rho \in \Pi$ :

$$
\begin{gathered}
\underline{\Delta A^{+}} \underline{x}^{+}-\overline{\Delta A} \underline{x}^{-}-\Delta A^{-} \bar{x}^{+}+\overline{\Delta A} \bar{x}^{-} \leq \Delta A(\rho) x \\
\leq \overline{\Delta A} \bar{x}^{+}-\underline{\Delta A^{+}} \bar{x}^{-}-\overline{\Delta A} \underline{x}^{+}+\underline{\Delta A^{-}} \underline{x}^{-}
\end{gathered}
$$

provided that $\underline{x} \leq x \leq \bar{x}$ for some $\underline{x}, \bar{x} \in \mathbb{R}^{n}$.

\subsection{Nonnegative LPV systems}

Let us start analysis with a simplified (but still widely met in applications) case of nonnegative system (4).

Assumption 3. $x(t) \in \mathbb{R}_{+}^{n}$ and $\underline{b}(t) \in \mathbb{R}_{+}^{n}$ for all $t \geq 0$; $\underline{\Delta A}=0$.

Under assumptions 1 and 3 we also have that $b(t), \bar{b}(t) \in$ $\mathbb{R}_{+}^{n}$ for all $t \geq 0$. Note that the condition $b(t) \in \mathbb{R}_{+}^{n}$ is required for the system (4) with $\Delta A(\rho(t)) \equiv 0$ to be nonnegative Farina and Rinaldi [2000]. The last condition $\Delta A=0$ simply means that $A_{0}$ is the minimal value of $A_{0}+$ $\overline{\Delta A}(\rho)$ for $\rho \in \Pi$ and $\overline{\Delta A} \geq 0$, this condition can be always satisfied under Assumption 2 and a suitable shift of $A_{0}$, $\triangle A$ and $\overline{\Delta A}$ (for nonnegative systems such a restriction simplifies the notations). Implicitly this assumption may imply that the matrix $A_{0}+\Delta A(\rho)$ is Metzler for all $\rho \in \Pi$.

Denote by $\underline{x}(t)$ and $\bar{x}(t)$ the lower and upper bound estimates of the state $x(t)$ respectively. Let us introduce two observer gain matrices $\underline{L}, \bar{L} \in \mathbb{R}^{n \times p}$, whose values will be specified later, then an interval observer structure for the nonnegative system (4) is given by:

$$
\begin{aligned}
\underline{\dot{x}}= & {\left[A_{0}-\underline{L} C\right] \underline{x} } \\
& +\max \left\{0, \underline{L} y-|\underline{L}| V E_{p}\right\}+\underline{b}(t), \\
\dot{\bar{x}}= & {\left[A_{0}-\bar{L} C+\overline{\Delta A}\right] \bar{x}+\bar{L} y+|\bar{L}| V E_{p}+\bar{b}(t) . }
\end{aligned}
$$

Note that it is a linear system, the conditions ensuring stability and interval estimation through (6) are stated below.

Theorem 4. Let assumptions 1-3 be satisfied, the matrices $A_{0}-\underline{L} C, A_{0}-\bar{L} C$ be Metzler and the following constraints be verified for some $\lambda_{1}, \lambda_{2} \in \mathbb{R}_{+}^{n} \backslash\{0\}$ :

$$
\begin{gathered}
{\left[A_{0}-\underline{L} C\right]^{\mathrm{T}} \lambda_{1}+Z^{\mathrm{T}} E_{s}<0,} \\
{\left[A_{0}-\bar{L} C+\overline{\Delta A}\right]^{\mathrm{T}} \lambda_{2}+Z^{\mathrm{T}} E_{s}<0,} \\
\lambda_{i}-\gamma E_{n}<0, i=1,2, \\
\underline{L} C \geq 0, \bar{L} C \geq 0
\end{gathered}
$$

for a scalar $\gamma>0$ and $Z \in \mathbb{R}_{+}^{s \times n}, 0<s \leq n$. Let $\underline{x}(0) \leq x(0) \leq \bar{x}(0)$, then the solutions of (4), (6) satisfy

$$
0 \leq \underline{x}(t) \leq x(t) \leq \bar{x}(t) \quad \forall t \geq 0
$$

and $\underline{x}, \bar{x} \in \mathcal{L}_{\infty}^{n}$. In addition the $L_{1}$ gain of the transfer functions $\underline{b} \rightarrow Z \underline{x}$ and $\bar{b} \rightarrow Z \bar{x}$ is less than $\gamma$.

All proofs are omitted for brevity of presentation.

The matrix $Z$ and the $L_{1}$ gain stability conditions are introduced in order to be able to improve/regulate the accuracy of interval estimation for some part of variables (for example, the matrix $Z$ can select all state coordinates excluding the measured variables). Note that the transfer functions $\underline{b} \rightarrow Z \underline{x}$ and $\bar{b} \rightarrow Z \bar{x}$ for (6) correspond to the transfer functions $b-\underline{b} \rightarrow Z \underline{e}$ and $\bar{b}-b \rightarrow Z \bar{e}$ for the estimation errors, thus the gain $\gamma$ really determines the interval estimation accuracy for (6). Recall that for a nonnegative system (6), existence of an $L_{1}$ gain implies existence of an $L_{\infty}$ gain Briat [2011], Ebihara et al. [2011].

Note that the conditions of Theorem 4 imposed on the gains $\underline{L}, \bar{L}$ can be formalized in terms of the following linear programming problem for the case $C \geq 0$ (see also Bolajraf et al. [2011]): it is required to find $\lambda_{1}, \lambda_{2} \in \mathbb{R}^{n}$ and $w_{1}, w_{2} \in \mathbb{R}_{+}^{p}\left(\lambda_{i}, w_{i}, i=1,2\right.$ are the decision variables $)$ such that

$$
\begin{gathered}
A_{0}^{\mathrm{T}} \lambda_{1}-C^{\mathrm{T}} w_{1}+Z^{\mathrm{T}} E_{s}<0, \\
{\left[A_{0}+\overline{\Delta A}\right]^{\mathrm{T}} \lambda_{2}-C^{\mathrm{T}} w_{2}+Z^{\mathrm{T}} E_{s}<0,} \\
\lambda_{i}-\gamma E_{n}<0, i=1,2 \\
\lambda_{i}>0, w_{i} \geq 0, i=1,2,
\end{gathered}
$$

then $\underline{L}, \bar{L} \in \mathbb{R}_{+}^{n \times p}$ are solutions of the equations $w_{1}=$ $\underline{L}^{\mathrm{T}} \lambda_{1}, w_{2}=\bar{L}^{\mathrm{T}} \lambda_{2}$. A possible approach is to select $\underline{L}=$ $\lambda_{1} w_{1}^{\mathrm{T}}\left|\lambda_{1}\right|^{-2}$ and $\bar{L}=\lambda_{2} w_{2}^{\mathrm{T}}\left|\lambda_{2}\right|^{-2}$. Thus the inequalities above have to be accompanied with constraints for a diagonal matrix $S \in \mathbb{R}_{+}^{n \times n}$ :

$$
\begin{gathered}
A_{0}-\underline{L} C+S \geq 0, A_{0}-\bar{L} C+S \geq 0, \\
\underline{L}=\lambda_{1} w_{1}^{\mathrm{T}}\left|\lambda_{1}\right|^{-2}, \bar{L}=\lambda_{2} w_{2}^{\mathrm{T}}\left|\lambda_{2}\right|^{-2} .
\end{gathered}
$$

The obtained set of inequalities can be rewritten as follows:

$$
\begin{gathered}
A_{0}^{\mathrm{T}} \lambda_{1}-C^{\mathrm{T}} w_{1}+Z^{\mathrm{T}} E_{s}<0, \\
{\left[A_{0}+\overline{\Delta A}\right]^{\mathrm{T}} \lambda_{2}-C^{\mathrm{T}} w_{2}+Z^{\mathrm{T}} E_{s}<0,} \\
\left|\lambda_{i}\right|=\gamma, \lambda_{i}>0, w_{i} \geq 0, i=1,2, \\
\underline{L}=\lambda_{1} w_{1}^{\mathrm{T}} \gamma^{-2}, \bar{L}=\lambda_{2} w_{2}^{\mathrm{T}} \gamma^{-2}, \\
A_{0}-\underline{L} C+S \geq 0, A_{0}-\bar{L} C+S \geq 0, S \geq 0,
\end{gathered}
$$

which can be resolved with respect to the variables $\lambda_{1}, \lambda_{2}$, $w_{1}, w_{2}$ and $S$ using an LMI toolbox.

The result of Theorem 4 can be useful for estimation in a large scale nano- or micro- system, which is described by the Chemical Master equation under the signal or parameter uncertainties Chen et al. [2012], Goh et al. [2010] (see an example of numerical simulations in Section 4).

Remark 5. The requirement that the matrices $A_{0}-\underline{L} C$, $A_{0}-\bar{L} C$ have to be Metzler can be relaxed by means of a change of coordinates $z=T x$ with a nonsingular matrix $T$ such that the matrices $T\left(A_{0}-\underline{L} C\right) T^{-1}, T\left(A_{0}-\bar{L} C\right) T^{-1}$ are Metzler. The matrix $T$ can be found using the results of Efimov et al. [2013a], Raïssi et al. [2012]. This extension is omitted for brevity of presentation. 


\subsection{Generic LPV systems}

For the case of non positive LPV system (4), the following interval observer structure is proposed:

$$
\begin{aligned}
\underline{\dot{x}}= & {\left[A_{0}-\underline{L} C\right] \underline{x}+\left[\underline{\Delta A^{+}} \underline{x}^{+}-\overline{\Delta A} \underline{x}^{-}\right.} \\
& \left.-\Delta A^{-} \bar{x}^{+}+\overline{\Delta A} \bar{x}^{-}\right]+\underline{L} y-|\underline{L}| V E_{p}+\underline{b}(t), \\
\dot{\bar{x}}= & {\left[A_{0}-\bar{L} C\right] \bar{x}+\left[\overline{\Delta A} \bar{x}^{+}-\Delta A^{+} \bar{x}^{-}\right.} \\
& \left.-\overline{\Delta A} \underline{x}^{+}+\underline{\Delta A^{-}} \underline{x}^{-}\right]+\bar{L} y+|\bar{L}| V E_{p}+\bar{b}(t) .
\end{aligned}
$$

Note that due to presence of $x^{+}, x^{-}, \bar{x}^{+}$and $\bar{x}^{-}$, the interval observer (8) is a globally Lipschitz nonlinear system.

Theorem 6. Let assumptions 1, 2 be satisfied and the matrices $A_{0}-\underline{L} C, A_{0}-\bar{L} C$ be Metzler. Then the relations (7) are satisfied provided that $x(0) \leq x(0) \leq \bar{x}(0)$. If the there exist $P \in \mathbb{R}^{2 n \times 2 n}, P=P^{\overline{\mathrm{T}}} \succ 0$ and $\gamma>0$ such that the following Riccati matrix inequality is verified

$$
G^{\mathrm{T}} P+P G+2 \gamma^{-2} P^{2}+4 \gamma^{2} \eta^{2} I_{2 n}+Z^{\mathrm{T}} Z \prec 0,
$$

where $\eta=\|\overline{\Delta A}-\underline{\Delta A}\|_{2}, Z \in \mathbb{R}^{s \times 2 n}, 0<s \leq 2 n$ and

$$
G=\left[\begin{array}{cc}
A_{0}-\underline{L} C+\underline{\Delta A^{+}} & -\underline{\Delta A^{-}} \\
-\overline{\Delta A}^{-} & A_{0}-\bar{L} C+\overline{\Delta A}
\end{array}\right],
$$

then $\underline{x}, \bar{x} \in \mathcal{L}_{\infty}^{n}$. In addition, the system (8) for the transfer $\left[\frac{b}{\bar{b}}\right] \rightarrow Z\left[\frac{x}{\bar{x}}\right]$ has an $L_{\infty}$ gain less than $\gamma$.

The Riccati matrix inequality from Theorem 6 can be reformulated in terms of LMIs with respect to $\underline{L}, \bar{L}$ and $P$. Indeed, $G=D-\Lambda \Upsilon$, where

$$
\begin{gathered}
D=\left[\begin{array}{cc}
A_{0}+\underline{\Delta A}^{+} & -\underline{\Delta A^{-}} \\
-\overline{\Delta A}^{-} & A_{0}+\overline{\Delta A}^{+}
\end{array}\right], \Lambda=\left[\begin{array}{cc}
\underline{L} & 0 \\
0 & \bar{L}
\end{array}\right], \\
\Upsilon=\left[\begin{array}{cc}
C & 0 \\
0 & C
\end{array}\right] .
\end{gathered}
$$

Then the Riccati inequality can be rewritten as follows:

$$
\begin{gathered}
D^{\mathrm{T}} P+P D-\Upsilon^{\mathrm{T}} W^{\mathrm{T}}-W \Upsilon \\
+2 \gamma^{-2} P^{2}+4 \gamma^{2} \eta^{2} I_{2 n}+Z^{\mathrm{T}} Z \prec 0,
\end{gathered}
$$

where $W=P \Lambda$ is a new variable. Using the Schur complement we obtain an equivalent LMI:

$$
\left[\begin{array}{cc}
0.5 \gamma^{2} I_{2 n} & P \\
P & \Upsilon^{\mathrm{T}} W^{\mathrm{T}}+W \Upsilon-D^{\mathrm{T}} P \\
& -P D-4 \gamma^{2} \eta^{2} I_{2 n}-Z^{\mathrm{T}} Z
\end{array}\right] \succ 0
$$

which has to be verified with a linear constraint (verification of Metzler property)

$$
P\left[\begin{array}{cc}
A_{0} & 0 \\
0 & A_{0}
\end{array}\right]-W \Upsilon+P S \geq 0
$$

for a sufficiently big diagonal matrix $S \in \mathbb{R}_{+}^{2 n \times 2 n}$ and an elementwise nonnegative $P$ (an additional restriction). The decision variables $P$ and $W$ also have to be declared block-diagonal. These linear inequalities can be solved using LMI toolboxes, as it is done in examples below.

\section{EXAMPLES}

In this section we consider two numerical examples to show validity of conditions of theorems 4 and 6 .

\subsection{Nonnegative droplet-based microfluidic system}

Following Chen et al. [2012], Goh et al. [2010] consider a model of droplet-based microfluidic system based on the Chemical Master equation:

$$
\begin{aligned}
& \dot{P}_{0}=-\kappa(t) P_{0}, P_{0}(0)=1, \\
& \dot{P}_{i}=\kappa(t)\left[P_{i-1}-P_{i}\right], P_{i}(0)=0, i=\overline{1, N},
\end{aligned}
$$

where $P_{i}, i=\overline{0, N}$ is the probability that a droplet contains $i$ crystals, and $\kappa(t) d t$ is the probability that a critical nucleus will form during an infinitesimal time interval $d t$. This model evaluates the crystal growth process in time. According to Chen et al. [2012] $\kappa(t)=J(S(t)) V(t)$, where $S(t)$ is the supersaturation and $V(t)$ is the droplet volume, both of them are assumed to be available from (noisy) measurements, but the function $J$ is not exactly known. Therefore, we will assume that for the function $\kappa(t)$ only a lower $\underline{\kappa}(t)$ and an upper $\bar{\kappa}(t)$ bounds are available. In addition, for simplicity of presentation we assume that the functions $\underline{\kappa}(t), \bar{\kappa}(t)$ are piecewise constant, i.e. there exist intervals $\left[t_{j}, t_{j+1}\right), j=0, K$ such that $\underline{\kappa}(t)=\underline{\kappa}_{j}, \bar{\kappa}(t)=\bar{\kappa}_{j}$ for all $t \in\left[t_{j}, t_{j+1}\right), t_{0}=0$. For this system there is no measurement of the state $(C=0)$.

Thus it is a time-varying autonomous linear system, but since the exact value of $\kappa(t)$ is not known, then the LPV framework has to be used. One of the main difficulties with this system is that the number of subsystems $N$ (the possible of number of crystals in a droplet) can be sufficiently large. And the only way to predict/evaluate a possible state of the crystal growth process in a droplet is based on estimation for Chemical Master equation.

The observer (6) on each interval $\left[t_{j}, t_{j+1}\right), j=0, K$ can be rewritten as follows:

$$
\begin{aligned}
& \underline{\dot{x}}=A_{0} \underline{x}, \\
& \dot{\bar{x}}=\left[A_{0}+\overline{\Delta A}\right] \bar{x},
\end{aligned}
$$

where

$$
\begin{aligned}
A_{0} & =\left[\begin{array}{ccccc}
-\bar{\kappa}_{j} & 0 & \ldots & 0 & 0 \\
\underline{\kappa}_{j} & -\bar{\kappa}_{j} & \ldots & 0 & 0 \\
\vdots & \vdots & \ddots & \vdots & \vdots \\
0 & 0 & \ldots & \underline{\kappa}_{j} & -\bar{\kappa}_{j}
\end{array}\right], \\
A_{0}+\overline{\Delta A} & =\left[\begin{array}{ccccc}
-\underline{\kappa}_{j} & 0 & \ldots & 0 & 0 \\
\bar{\kappa}_{j} & -\underline{\kappa}_{j} & \ldots & 0 & 0 \\
\vdots & \vdots & \ddots & \vdots & \vdots \\
0 & 0 & \ldots & \bar{\kappa}_{j} & -\underline{\kappa}_{j}
\end{array}\right]
\end{aligned}
$$

and all conditions of Theorem 4 are satisfied for $\underline{L}=\bar{L}=0$ (no measurements). Thus we can iteratively apply the obtained interval observer (6) on each interval $\left[t_{j}, t_{j+1}\right), j=$ $0, K$ in order to reconstruct the distribution $P_{i}\left(t_{K+1}\right)$, $i=\overline{0, N}$ at the end the process of crystallization, starting from a fixed initial distribution $\left(P_{0}(0)=1\right.$ and $P_{i}(0)=0$ for $i=\overline{1, N})$.

For $\underline{\kappa}(t), \bar{\kappa}(t)$ given in Fig 1 and $N=32$ the results of interval estimation of $P_{i}\left(t_{K+1}\right), i=\overline{0, N}$ are shown in Fig 1. As we can conclude from these results, even a small difference in $\underline{\kappa}(t), \bar{\kappa}(t)$ may lead on a short time interval 

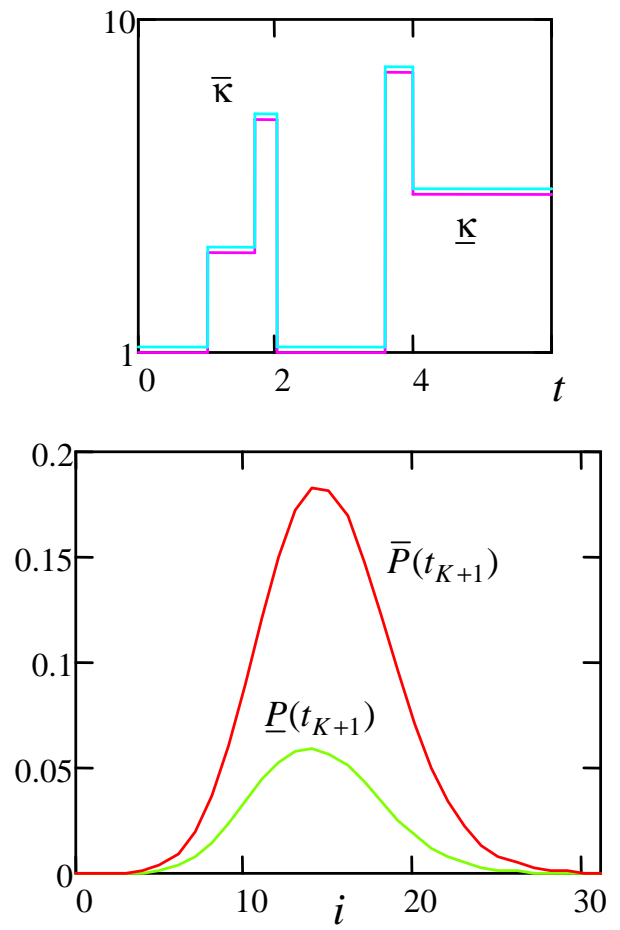

Fig. 1. The results of simulations for a nonnegative microfluidic system

$\left(t_{K+1}=6\right)$ to a big deviations of $\underline{P}_{i}\left(t_{K+1}\right)$ and $\bar{P}_{i}\left(t_{K+1}\right)$, $i=\overline{0, N}$.

\subsection{An academic LPV system}

Consider a nonlinear system:

$$
\begin{aligned}
\dot{x}= & {\left[\begin{array}{ccc}
\epsilon \cos t & 1+\epsilon \sin x_{3} & \varepsilon \sin x_{2} \\
\varepsilon \sin x_{3} & -0.5+\epsilon \sin t & 1+\epsilon \cos 2 t \\
\varepsilon \sin x_{2} & 0.3+\varepsilon \cos 2 t & -1+\epsilon \sin t
\end{array}\right] x } \\
& +\left[\begin{array}{c}
6 \cos x_{1} \\
\sin t+0.1 \sin x_{3} \\
-\cos 3 t+0.1 \sin 2 x_{2}
\end{array}\right], y=x_{1}+v(t),
\end{aligned}
$$

where $\epsilon=0.01$ and $\varepsilon=0.001$. We assume that $V=0.1$, and for simulation we selected $v(t)=V(\sin 5 t+\cos 3 t) / 2$. For initial conditions $\left|x_{i}(0)\right| \leq 5$ the system has bounded solutions. This system can be presented in the form of (4) for

$$
\begin{aligned}
& A_{0}=\left[\begin{array}{ccc}
0 & 1 & 0 \\
0 & -0.5 & 1 \\
0 & 0.3 & -1
\end{array}\right], \overline{\Delta A}=\left[\begin{array}{ccc}
\epsilon & \epsilon & \varepsilon \\
\varepsilon & \epsilon & \epsilon \\
\varepsilon & \varepsilon & \epsilon
\end{array}\right]=-\underline{\Delta A}, \\
& \underline{b}(t, y)=\left[\begin{array}{c}
6 f(y) \\
\sin \bar{t}-0.1 \\
-\cos 3 t-0.1
\end{array}\right], \bar{b}(t, y)=\left[\begin{array}{c}
6 \bar{f}(y) \\
\sin t+0.1 \\
-\cos 3 t+0.1
\end{array}\right] \text {, } \\
& \underline{f}(y)=\left\{\begin{array}{ll}
\cos y \cos V & \text { if } \cos y \geq 0 \\
\cos y & \text { if } \cos y<0
\end{array}-|\sin y| \sin V,\right. \\
& \bar{f}(y)=\left\{\begin{array}{ll}
\cos y & \text { if } \cos y \geq 0 \\
\cos y \cos V & \text { if } \cos y<0
\end{array}+|\sin y| \sin V\right.
\end{aligned}
$$

and a properly selected $\rho$, clearly assumptions 1 and 2 are satisfied. The LMIs formulated after Theorem 6 give a solution
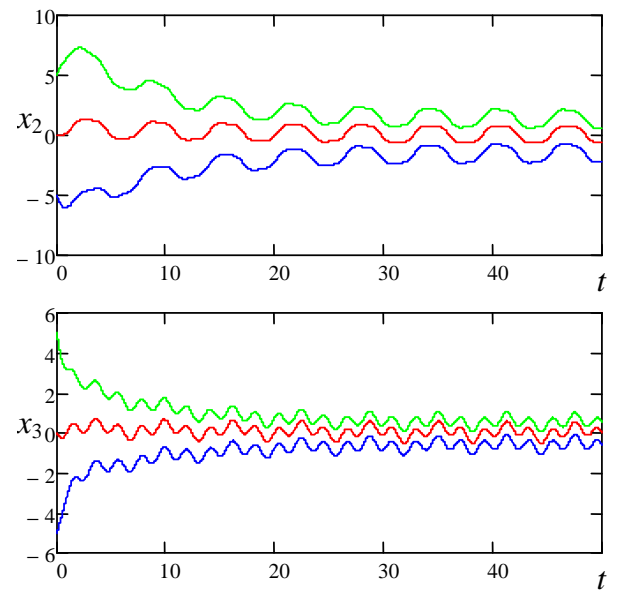

Fig. 2. The results of simulations for an LPV system

$$
\underline{L}=\left[\begin{array}{c}
77.97 \\
-0.07 \\
-0.11
\end{array}\right], \bar{L}=\left[\begin{array}{l}
77.75 \\
-0.02 \\
-0.04
\end{array}\right]
$$

for $\gamma=40$ (YALMIP toolbox has been used) and the matrix $Z$ selecting the variables $x_{2}$ and $x_{3}$. The results of interval simulations the variables $x_{2}$ and $x_{3}$ are given in Fig. 2.

\section{CONCLUSION}

This paper is devoted to design of interval observers for LPV systems with unmeasurable vector of scheduling parameters. Two cases are considered: general continuoustime LPV systems and nonnegative ones. For both cases interval observers are proposed, their cooperativity and stability are expressed in terms of matrix inequalities (nonlinear in a common case). It is shown that under some additional mild restrictions these inequalities can be represented in a form suitable for application of numerical solvers. Efficiency of the proposed observers is demonstrated on numerical simulations. Reducing the conservatism of the proposed LMIs and interval observers is a direction of future researches.

\section{REFERENCES}

G. Besanon, editor. Nonlinear Observers and Applications, volume 363 of Lecture Notes in Control and Information Sciences. Springer, 2007.

Mohamed Bolajraf, Mustapha Ait Rami, and Uwe R. Helmke. Robust positive interval observers for uncertain positive systems. In Proc. of the 18th IFAC World Congress, pages 14330-14334, 2011.

C. Briat. Robust stability analysis of uncertain linear positive systems via integral linear constraints: L1- and linfty-gain characterizations. In Proc. 50th IEEE CDC and ECC, pages 6337-6342, Orlando, 2011.

K. Chen, L.M. Goh, G.W. He, V. Bhamidi, P.J.A. Kenis, C.F. Zukoski, and Braatz R.D. Identification of nucleation rates in droplet-based microfluidic systems. Chem. Eng. Sci., 77:235-241, 2012.

C. Combastel. Stable interval observers in c for linear systems with time-varying input bounds. Automatic Control, IEEE Transactions on, $\mathrm{PP}(99)$ :1-6, 2013. ISSN 0018-9286. doi: 10.1109/TAC.2012.2208291. 
Y. Ebihara, D. Peaucelle, and D. Arzelier. L1 gain analysis of linear positive systems and its application. In Proc. 50th IEEE CDC and ECC, pages 4029-4035, Orlando, 2011.

D. Efimov, L.M. Fridman, T. Raïssi, A. Zolghadri, and R. Seydou. Interval estimation for lpv systems applying high order sliding mode techniques. Automatica, 48: 2365-2371, 2012.

D. Efimov, T. Raïssi, S. Chebotarev, and A. Zolghadri. Interval state observer for nonlinear time varying systems. Automatica, 49(1):200-205, 2013a.

D. Efimov, T. Raïssi, and A. Zolghadri. Control of nonlinear and lpv systems: interval observer-based framework. IEEE Trans. Automatic Control, 58(3):773-782, 2013b.

L. Farina and S. Rinaldi. Positive Linear Systems: Theory and Applications. Wiley, New York, 2000.

T.I. Fossen and H. Nijmeijer. New Directions in Nonlinear Observer Design. Springer, 1999.

Limay Goh, Kejia Chen, Venkateswarlu Bhamidi, Guangwen He, Nicholas C. S. Kee, Paul J. A. Kenis, Charles F. Zukoski, and Richard D. Braatz. A stochastic model for nucleation kinetics determination in droplet-based microfluidic systems. Crystal Growth \& Design, 10 (6):2515-2521, 2010. doi: $10.1021 / \operatorname{cg} 900830 y$. URL http://pubs.acs.org/doi/abs/10.1021/cg900830y.

L. Jaulin. Nonlinear bounded-error state estimation of continuous time systems. Automatica, 38(2):1079-1082, 2002.

M. Kieffer and E. Walter. Guaranteed nonlinear state estimator for cooperative systems. Numerical Algorithms, $37: 187-198,2004$

A. Marcos and J. Balas. Development of linear-parametervarying models for aircraft. J. Guidance, Control, Dynamics, 27(2):218-228, 2004.

F. Mazenc and O. Bernard. Interval observers for linear time-invariant systems with disturbances. Automatica, 47(1):140-147, 2011

T. Meurer, K. Graichen, and E.-D. Gilles, editors. Control and Observer Design for Nonlinear Finite and Infinite Dimensional Systems, volume 322 of Lecture Notes in Control and Information Sciences. Springer, 2005.

M. Moisan, O. Bernard, and J.L. Gouzé. Near optimal interval observers bundle for uncertain bio-reactors. Automatica, 45(1):291-295, 2009.

B. Olivier and J.L. Gouzé. Closed loop observers bundle for uncertain biotechnological models. Journal of Process Control, 14(7):765-774, 2004. ISSN 0959-1524.

T. Raïssi, G. Videau, and A. Zolghadri. Interval observers design for consistency checks of nonlinear continuoustime systems. Automatica, 46(3):518-527, 2010.

T. Raïssi, D. Efimov, and A. Zolghadri. Interval state estimation for a class of nonlinear systems. IEEE Trans. Automatic Control, 57(1):260-265, 2012.

J. Shamma and J. Cloutier. Gain-scheduled missile autopilot design using linear parameter-varying transformations. J. Guidance, Control, Dynamics, 16(2):256261, 1993.

J.S. Shamma. Control of Linear Parameter Varying Systems with Applications, chapter An overview of LPV systems, pages 1-22. Springer, 2012.

H.L. Smith. Monotone Dynamical Systems: An Introduction to the Theory of Competitive and Cooperative Systems, volume 41 of Surveys and Monographs. AMS,
Providence, 1995.

W. Tan. Applications of Linear Parameter-Varying Control Theory. PhD thesis, Dept. of Mechanical Engineering, University of California at Berkeley, 1997. 